\section{Aerobic exercise reduces depressive symptoms in patients with chronic heart failure}

doi:10.1136/eb-2012-101036

\section{QUESTION}

Question: Does exercise training reduce depressive symptoms in patients with chronic heart failure compared with guideline-based usual care?

Patients: 2322 Stable patients with chronic heart failure participating in the Heart Failure-A Controlled Trail Investigating Outcomes of Exercise Training (HF-ACTION) trial who completed the Beck Depression Inventory II (BDI-II) at baseline. Possible BDI-II scores range from 0 to 63 ; scores of $\geq 14$ were considered clinically significant depressive symptoms. Among participants, BDI-II scores ranged from 0 to 59 (median 8), and $28 \%$ had clinically significant depressive symptoms.

Setting: 82 Medical centres in the USA, Canada and France; April 2003 to February 2007.

Intervention: Aerobic exercise versus usual care alone. Participants randomised to aerobic exercise were provided with a treadmill or exercise bicycle and received three supervised exercise sessions per week for 3 months (90 min/week), transitioning to home-based unsupervised exercise (goal $120 \mathrm{~min} /$ week) for the next 9 months. Usual care participants were not given a formal exercise prescription. All participants received self-management educational materials and regular follow-up phone calls.

Outcomes: Change in depressive symptoms after 3 months exercise (primary outcome, end of the supervised exercise period), and after 12 months (secondary outcome); all-cause mortality, hospitalisation or both (primary medical outcomes); cardiovascular and heart failure hospitalisations and mortality (secondary medical outcomes).

Patient follow-up: $99.6 \%$ Included in primary analyses.

\section{METHODS}

Design: Randomised controlled trial.

Allocation: Concealed.

Blinding: Single blind for clinical events (assessor-blinded).

Follow-up period: Median 30 months.

\section{MAIN RESULTS}

After 3 and 12 months, aerobic exercise significantly reduced depressive symptoms compared with usual care alone (adjusted mean BDI-II score at 3 months: 8.95 with exercise vs 9.70 with usual care, $p=0.002$; at 12 months: 8.86 with exercise vs 9.54 with usual care group, $\mathrm{p}=0.01$ ). Among the subgroup of patients with clinically significant depressive symptoms, aerobic exercise also significantly reduced depressive symptoms compared with usual care alone at both timepoints (mean BDI-II score at 3 months: 16.66 with exercise vs 17.98 with usual care, $p=0.04$; at 12 months: 15.85 with exercise vs 17.34 with usual care, $p=0.02$ ). When compared to usual care, aerobic exercise was associated with a lower risk of all-cause death or hospitalisation (HR $0.89,95 \%$ CI 0.81 to 0.99 , $\mathrm{p}=0.03$ ), and a lower risk of heart failure hospitalisations and deaths (HR $0.85,95 \%$ CI 0.73 to $0.98, p=0.03$ ). There was no significant effect of aerobic exercise on cardiovascular hospitalisations and deaths (HR 0.91, 95\% CI 0.81 to 1.01, p=0.09). Higher baseline BDI-II was associated with an increased risk of clinical events (HR for an event in participants with BDI-II score $\geq 14$ vs participants with a score of $4: 1.16,95 \%$ CI 1.05 to $1.29, \mathrm{p}=0.01$ ). After adjustment for covariates, there was a significant association between change in BDI-II score and risk of all cause death or hospitalisation, with improvement linked to reduced risk of an event $(p=0.02)$.

\section{CONCLUSIONS}

Aerobic exercise produces a small reduction in depressive symptoms in patients with heart failure compared to usual care alone.

\section{NOTES}

Only $40 \%$ of patients in the aerobic exercise group were fully adherent to the exercise programme.

\section{ABSTRACTED FROM}

Blumenthal JA, Babyak MA, O'Connor $\mathrm{C}$, et al. Effects of exercise training on depressive symptoms in patients with chronic heart failure: the HF-ACTION randomized trial. JAMA 2012;308:465-74.

Correspondence to: James A Blumenthal, Department of Psychiatry and Behavioural Sciences, Duke University Medical Center, PO Box 3119, Durham, NC 27710, USA; blume003@mc.duke.edu

Sources of funding: The US National Heart, Lung and Blood Institute.

- Additional material is published online only. To view please visit the journal online (http://dx.doi.org/10.1136/eb-2012-101036). n this ancillary study of the Heart Failure-A Controlled Trial Investigating Outcomes of Exercise Training (HF-ACTION) by Blumenthal and colleagues, the effects of aerobic exercise on depressive symptoms was examined using the Beck Depression Inventory-ll (BDI-II) and clinical outcomes at 3 and 12 months in persons with heart failure (HF). Exercise had a sustainable effect on depressive symptom reduction at 3 and 12 months compared to usual care controls. Lower BDI scores were also associated with fewer adverse events and hospitalisations; the benefit of exercise was more pronounced with $\mathrm{BDI}$ scores $>14$, consistent with a previous report. The findings from this study support previous research of the benefits of exercise on depressive symptoms in $\mathrm{HF}^{1}$ and also suggests a protective benefit that may attenuate adverse clinical outcomes associated with subclinical depression.

Current HF guidelines recommend moderate levels of continuous aerobic and resistance exercise to optimise health outcomes. The majority of HF patients, however, are non-adherent to exercise, despite being advised to do so or being aware of its positive health benefits; the presence of depressive symptoms causes a further reduction in adherence. Exercise adherence was low $(<43 \%)$ across all time points in the HF-ACTION study. ${ }^{2}$ Factors that contributed to high or low levels of exercise adherence among participants was not provided, but may provide important insights for future research. Additionally, the supervised and home-based components of the intervention had a similar influence on outcomes. Questions about which settings and strategies promote greater adherence are of paramount importance, especially since less than $30 \%$ of eligible persons attend cardiac rehabilitation programmes, and attrition rates are high. Home-based strategies may provide a cost-effective and practical approach to increase exercise participation in persons with $\mathrm{HF}$ and warrants further investigation. Finally, the extent to which HF-ACTION ${ }^{2}$ and other exercise studies translate to the general HF population is unclear since most have been conducted in highly motivated, adherent, younger Caucasian males with systolic heart failure. Future studies are needed that more accurately depict the general HF population - older people with multiple comorbidities, and $40-50 \%$ female with preserved systolic function.

\section{Rebecca A Gary}

School of Nursing, Emory University, Atlanta, Georgia, USA Competing interests None.

\section{REFERENCES}

1. Gary RA, Dunbar SB, Higgins MK, et al. Combined exercise and cognitive behavioral therapy improves outcomes in patients with heart failure. $J$ Psychosomatic Res 2010;69:119-31.

2. O'Connor CM, Whellan DJ, Lee $\mathrm{KL}$, et al. Efficacy and safety of exercise training in patients with chronic heart failure: HF-ACTION randomized controlled trial. JAMA 2009;301:1439-50. 